\title{
One-dimensional steady-state photorefractive spatial solitons in centrosymmetric paraelectric potassium lithium tantalate niobate
}

\author{
Eugenio DelRe, Bruno Crosignani, and Mario Tamburrini \\ Dipartimento di Fisica, Università degli Sudi dell'Aquila, L'Aquila, Italy, and Fondazione Ugo Bordoni, Rome, Italy
}

Mordechai Segev and Matthew Mitchell

Department of Electrical Engineering, Princeton University, Princeton, New Jersey, 08544-5263

Eli Refaeli and Aharon J. Agranat

Department of Applied Physics, Hebrew University, Jerusalem 91904, Israel

Received October 24, 1997

\begin{abstract}
We report the first observation of spatial one-dimensional photorefractive screening solitons in centrosymmetric media and compare the experimental results with recent theoretical predictions. We find good qualitative agreement with theory. (c) 1998 Optical Society of America

OCIS codes: $190.5330,230.6120$.
\end{abstract}

Photorefractive spatial solitons have been a subject of intense study over the past few years. They have been predicted and observed in the quasi-steady-state regime ${ }^{1,2}$ in photovoltaic materials, ${ }^{3,4}$ in the screening configuration, ${ }^{5-9}$ and in photorefractive semiconductors. ${ }^{10}$ More-complicated phenomena have also been reported, giving rise to intriguing observations, such as self-trapping of incoherent light beams. ${ }^{11}$ All these phenomena have been observed in noncentrosymmetric materials, in which soliton formation is governed by a change in refractive index that is due to the linear electro-optic response to an internal photoinduced space-charge field. Recently, spatial screening solitons of a different nature that should exist in photorefractive centrosymmetric materials were predicted. ${ }^{12}$ We report the first observation of such solitons and compare our experimental results with the theoretical predictions.

Centrosymmetric screening solitons arise from the index change produced by the quadratic electro-optic response to a photoinduced internal field. The field has, in this case, the double role both of polarizing the crystalline structure and of distorting the electronic polarization. In centrosymmetric crystals the change in refractive index is proportional to the square of the polarization $(1 / \Delta n)_{i j}=g_{i j k l} P_{k} P_{l}$ and is expressed by $\Delta n=-(1 / 2) n_{b}{ }^{3} g_{\text {eff }} \epsilon_{0}^{2}\left(\epsilon_{r}-1\right)^{2} E^{2}$, where $E$ is the internal field, $g_{\text {eff }}$ is the effective quadratic electrooptic coefficient, and $n_{b}$ is the background refractive index and it is assumed that the (dc) polarization is in the linear regime, i.e., $P=\epsilon_{0}\left(\epsilon_{r}-1\right) E$.

Our experiments are performed in potassium lithium tantalate niobate ${ }^{13}$ (KLTN), which is treated to have a first-order ferroelectric-paraelectric phase transition slightly below room temperature. Working at room temperature enables one to operate in a centrosymmetric phase close to that transition, thereby enhancing the electro-optic response,$^{13}$ making centrosymmetric soliton observation possible with moderate electric fields. In Fig. 1 we show $\epsilon_{r}$ as a function of temperature and observe the large increase of $\epsilon_{r}$ at the vicinity of the ferroelectric-paraelectric transition (which occurs at $\sim 12{ }^{\circ} \mathrm{C}$ ). Because $\Delta n$ scales with $\left(\epsilon_{r}-1\right)^{2}$, operation at temperatures slightly above the Curie temperature results in an increase of the quadratic electro-optic response. In the specific case of KLTN, $g_{\text {eff }}$ is positive and thus only bright solitons can be observed; i.e., in the screening regime KLTN is a self-focusing medium. ${ }^{12}$

Bright centrosymmetric screening solitons in $(1+1)$ $D$ obey the wave equation

$$
\frac{\mathrm{d}^{2} u(\xi)}{\mathrm{d} \xi^{2}}=-\left\{\frac{1}{1+u_{0}^{2}}-\frac{1}{\left[1+u^{2}(\xi)\right]^{2}}\right\} u(\xi),
$$

where $u(\xi)$ is the soliton amplitude normalized to the square root of the sum of background and dark irradiances; $\xi=x / d$ is the transverse coordinate normalized to $d=(-2 k b)^{-1 / 2}$, with $b=\left(k / n_{b}\right)(1 / 2) n_{b}{ }^{3} g_{\text {eff }} \epsilon_{0}^{2}\left(\epsilon_{r}-\right.$ $1)^{2}(V / L)^{2} ; k=2 \pi n_{b} / \lambda ; \lambda$ is the vacuum wavelength; $V$ is the applied voltage; and $L$ is the width of the crystal between the electrodes, which, for $(1+1) D$ solitons, is also the narrow dimension of the beam, as shown

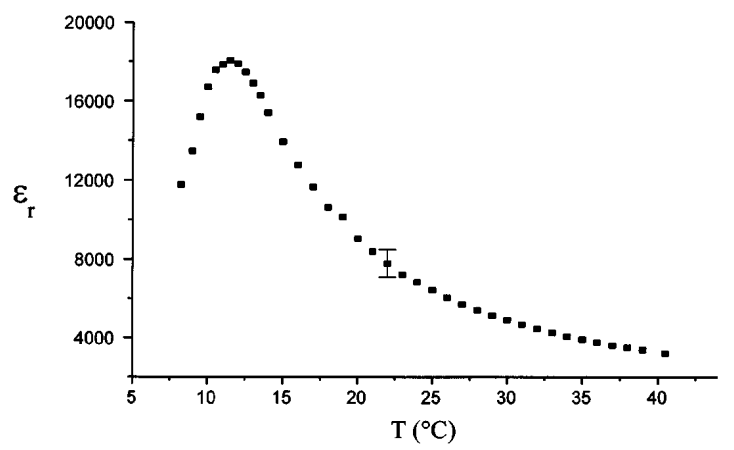

Fig. 1. Measured values of low frequency $\epsilon_{r}$ as a function of temperature $T$ in our KLTN sample. The error bar indicates the experimental error. 
in Fig. 2. Solutions of Eq. (1) form a set of parameter values that support soliton formation. For a given value of $u_{0}$, only one value of intensity FWHM (in units of $\xi$ ) can give rise to self-trapping. ${ }^{12}$ This relation is often referred to as the soliton existence curve.

Figure 2 shows the experimental setup. A cw argon-ion laser beam is split into two orthogonal polarizations by a polarizing beam splitter. The transmitted beam, polarized parallel to the plane $(x$ axis) of the figure, is focused by a cylindrical lens onto the input face of the KLTN crystal, with its narrow dimension parallel to $x$. The crystal is of dimensions $3.7 \mathrm{~mm} \times 4.6 \mathrm{~mm} \times 2.4 \mathrm{~mm}$ in the $\hat{x}, \hat{y}$, and $\hat{z}$ directions, where $\hat{z}$ is the direction of propagation. The sample is kept at a constant temperature by a current-controlled Peltier junction. We measured the effective quadratic electro-optic coefficient (in a separate electro-optic-interferometric experiment) and found that $g_{\text {eff }}=0.12 \mathrm{~m}^{4} \mathrm{C}^{-2}$ and $n_{b}=2.2$. Gold electrodes are sputtered onto the $\hat{x}$-axis faces, to which an external voltage $V$ is applied. Finally, we image the input and output faces of the sample onto the sensitive area of a CCD camera. The $\hat{y}$-axis polarized beam serves as the background beam: It is expanded, recombined with the soliton beam, and made to illuminate the crystal uniformly while copropagating with the soliton-forming beam.

In Fig. 3 we show typical experimental results: photographs and beam profiles at the input face of the crystal (left) and at the output face in the normal diffraction regime (middle; zero voltage). A one-dimensional soliton forms with the application of an appropriate voltage (right). In the particular case shown in Fig. 3 the input beam is $9 \mu \mathrm{m}$ FWHM, and it diffracts to $29 \mu \mathrm{m}$ with $V=0$. The self-trapped (soliton) beam has the same width as the input beam, $9 \mu \mathrm{m}$, and it forms with intensity ratio $u_{0}{ }^{2} \cong 2.9$, $V \cong 2 \mathrm{kV}$, and a sample temperature of $T=21^{\circ} \mathrm{C}$.

To compare our experimental results with the theory ${ }^{12}$ we performed a number of experiments. First we note that the choice of the crystalline direction along which we apply the external field is fully arbitrary as long as the field is parallel to the direction along which the intensity profile of the soliton varies. This means that having the input one-dimensional beam narrow in the $x$ direction and by applying an external field in the $x$ direction, one can rotate the crystal and launch the soliton in any direction. This is manifested in our experiments by the fact that we have chosen the surfaces covered with electrodes (normal to $x$ ) arbitrarily in the crystal preparation process. Second, once we establish the soliton, we reverse the polarity of the applied field and adjust the voltage until the soliton forms at the opposite polarity. As $\Delta n$ depends on $E^{2}$, the soliton should be insensitive to polarity reversal of $E$. In our experiments the soliton is indeed reestablished at reversed polarity, but it typically requires a somewhat higher voltage $(\sim 100-\mathrm{V}$ shift for the result shown in Fig. 3), an $\sim 5 \%$ difference between the two applied field polarities. The ability to generate the same soliton with either positive or negative field polarities is unique to photorefractive solitons that are driven by the dc Kerr effect and proves that our observation is indeed of photorefractive solitons in centrosymmetric media. This is in sharp contradistinction to photorefractive solitons in noncentrosymmetric media, which are all driven by the Pockels effect, for which one polarity gives rise to bright solitons only whereas the reverse polarity supports dark solitons only. Here the shift between the field values applied in both polarities is attributed to the formation of a dc space-charge field, which causes the internal field to be slightly shifted with respect to the applied field (the strength of the space-charge field is orientation dependent owing to the nonuniformity of the crystal formed during the crystal growth). A similar phenomenon was observed in formation of regular gratings at the paraelectric phase..$^{14}$

Finally, we investigated experimentally the soliton existence curve and compared it with the prediction. Keeping the input beam fixed, we varied the intensity ratio and determined at which voltage $V$ steady-state solitons occur. Knowing the (measured) sample temperature $T$, we find the values of $V$ necessary for soliton solutions, given a value of $u_{0}$. By plotting the experimental points against the predicted existence curve we are able to assess the adherence of the theory to experiments. Figure 4 compares the theoretical existence curve with the measured values for two temperatures (at which $\epsilon$ and $g_{\text {eff }}$ attain different values). Notice that, for values of $u_{0}>1.5$, the normalized width $(\Delta \xi)$, which is proportional to the applied voltage $V$, has a linear dependence on $u_{0}$, which is observed in both the theoretical and the experimental results (although the slopes are somewhat different). This dependence is

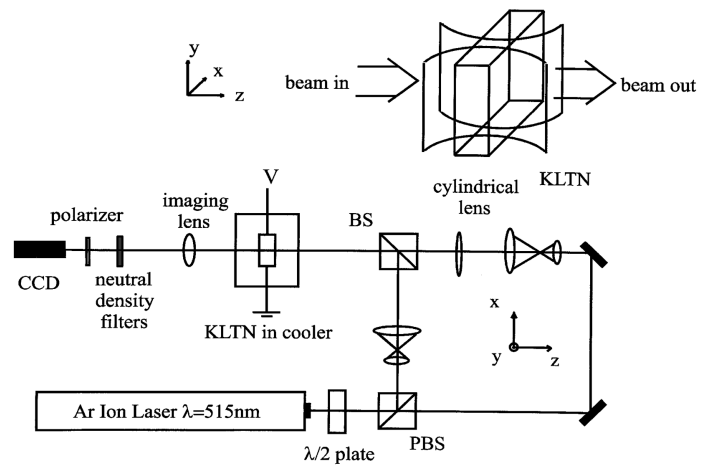

Fig. 2. Experimental setup: BS, beam splitter; PBS, polarizing beam splitter.

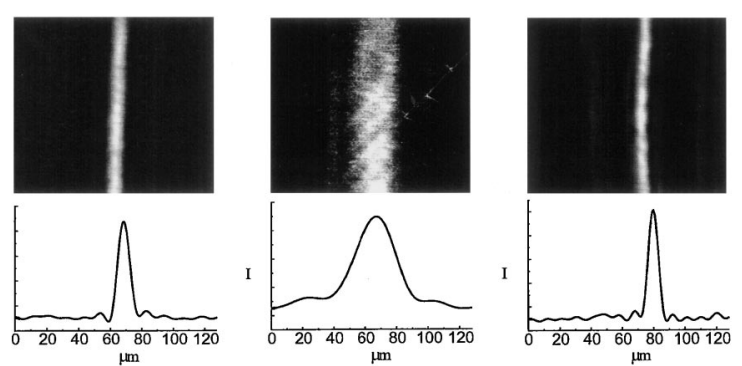

Fig. 3. Photographs and profiles of the 9- $\mu \mathrm{m}$ FWHM input beam (left), the diffracted output beam at $V=0$ (middle), and the soliton output (right). The profiles are normalized to their maximum values. 


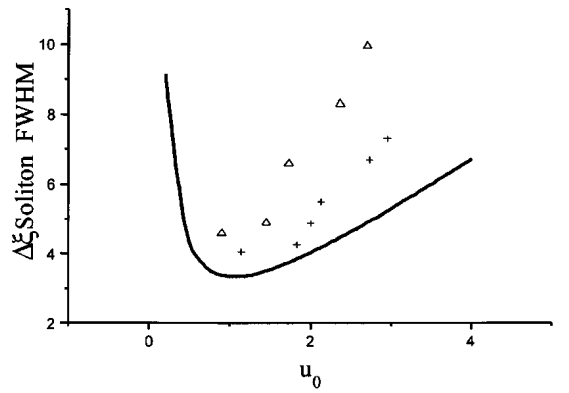

Fig. 4. Soliton existence curves: theoretically predicted (solid curve) and experimentally measured points at $18.5^{\circ} \mathrm{C}$ (crosses) and at $21^{\circ} \mathrm{C}$ (triangles).

unique to this type of soliton and as opposed to the dependence of $V$ on $u_{0}$ for screening solitons that rely on the linear electro-optic effect [in that case $\Delta \xi \propto \sqrt{V}$ and at high intensity ratios $\Delta \xi \propto u_{0}$ (Ref. 7)]. This result confirms that these solitons indeed rely on the quadratic electro-optic effect. Another observation is that for both temperatures the existence curve flattens near $u_{0}=1$, consistent with the theory.

As is apparent from Fig. 4, although there is good qualitative agreement between theory and experiments the experimental values are shifted from the theoretical curve (for $u_{0}>2$ ) and the curves for both temperatures do not fully overlap (as expected from Ref. 12). There are several plausible reasons for this discrepancy. The primary reason is that in the theory the background illumination is assumed to be uniform in $x$, whereas in practice this beam is slightly guided under the soliton. In other words, the spacecharge field generated by the soliton gives rise to $\Delta n$ not only for the $\hat{x}$-polarized (soliton) beam (through $g_{i i i i}=g_{x x x x}$ ), as it should, but also for the $\hat{y}$-polarized (background) beam (through $g_{j j i i}=g_{y y x x}$ ), causing the background beam to be slightly guided in the region of the soliton. We observed this weak guidance of the background beam under the soliton in all our experiments (by rotating the polarizer in front of the camera). Similar effects were observed in all experiments with screening solitons that rely on the Pockels effect, and they always led to a shift of the measured existence curve to higher $\Delta \xi$ values. ${ }^{15}$ In the present case these effects are even more pronounced [than for ferroelectric crystals such as strontium barium niobate $(\mathrm{SBN})]$, because in ideally isotropic media $g_{j j i i} / g_{i i i i}=$ $2 / 3$, compared with $r_{13} / r_{33} \approx 1 / 6$ in SBN:60. This result can explain the deviation of the experimental existence curve from the theoretical prediction but cannot explain why the two data sets at the different temperatures also deviate from each other. A plausible explanation is that, at the proximity of the phase transition, $\epsilon_{r}$ depends on the local field. ${ }^{16}$ This means that the quadratic electro-optic effect deviates slightly from quadratic dependence on $E$, implying that, in these materials, interesting phase-transition-related phenomena play an important role in soliton formation. We expect that, at temperatures that are even closer to the phase transition, other phenomena such as critical slowing, hysteresis, and fixing ${ }^{17}$ will become important, and the soliton itself can prove to be a sensitive means of investigation. For applications, fixing (by cooling through the Curie temperature ${ }^{17}$ ) will permit highly versatile imprinting of complicated optical circuitry, making bulk optical reconfigurable waveguide components possible. We also expect these materials to support $(2+1) D$ spatial solitons, as the ferroelectric photorefractive materials (e.g., SBN) do.

In conclusion, we have reported the first observation of spatial solitons in a photorefractive centrosymmetric medium.

The research of $M$. Tamburrini was carried out in the framework of an agreement between the Fondazione Bordoni and the Italian Communications Administration. B. Crosignani gratefully acknowledges the support of collaborative NATO research grant 930240. The research of M. Mitchell and M. Segev was supported by the National Science Foundation. That of A. Agranat and E. Refaeli was supported by the Ministry of Science of the State of Israel.

\section{References}

1. M. Segev, B. Crosignani, A. Yariv, and B. Fischer, Phys. Rev. Lett. 68, 923 (1992).

2. G. Duree, J. L. Shultz, G. Salamo, M. Segev, A. Yariv, B. Crosignani, P. DiPorto, E. Sharp, and R. Neurgaonkar, Phys. Rev. Lett. 71, 533 (1993).

3. G. C. Valley, M. Segev, B. Crosignani, A. Yariv, M. M. Fejer, and M. Bashaw, Phys. Rev. A 50, R4457 (1994).

4. M. Taya, M. Bashaw, M. M. Fejer, M. Segev, and G. C. Valley, Phys. Rev. A 52, 3095 (1995).

5. M. Segev, G. C. Valley, B. Crosignani, P. DiPorto, and A. Yariv, Phys. Rev. Lett. 73, 3211 (1994).

6. D. N. Christodoulides and M. I. Carvalho, J. Opt. Soc. Am. B 12, 1628 (1995); Opt. Commun. 118, 569 (1995).

7. M. Segev, M. Shih, and G. C. Valley, J. Opt. Soc. Am. B 13, 706 (1996).

8. M. D. Iturbe-Castillo, P. A. Marquez-Aguilar, J. J. Sánchez-Mondragón, S. Stepanov, and V. Vysloukh, Appl. Phys. Lett. 64, 408 (1994).

9. M. Shih, M. Segev, G. C. Valley, G. Salamo, B. Crosignani, and P. DiPorto, Electron. Lett. 31, 826 (1995); M. Shih, P. Leach, M. Segev, M. H. Garrett, G. Salamo, and G. C. Valley, Opt. Lett. 21, 324 (1996).

10. M. Chauvet, S. A. Hawkins, G. Salamo, M. Segev, D. F. Bliss, and G. Bryant, Opt. Lett. 21, 1333 (1996).

11. M. Mitchell and M. Segev, Nature (London) 387, 880 (1997).

12. M. Segev and A. Agranat, Opt. Lett. 22, 1299 (1997).

13. A. Agranat, R. Hofmeister, and A. Yariv, Opt. Lett. 17, 713 (1992).

14. A. J. Agranat, M. Razvag, M. Balberg, and V. Leyva, J. Opt. Soc. Am. B 14, 2043 (1997).

15. See, e.g., K. Kos, H. Ming, G. Salamo, M. Shih, M. Segev, and G. C. Valley, Phys. Rev. E 53, R4330 (1996).

16. R. Blinc, Soft modes of Ferroelectrics and Antiferroelectrics (North-Holland, Amsterdam, 1974), Chap. 3.

17. X. Tong, R. Hofmeister, M. Zhang, A. Yariv, A. Agranat, and V. Leyva, Opt. Lett. 21, 1860 (1996); A. J. Agranat, M. Razvag, and M. Balberg, Appl. Phys. Lett. 68, 2469 (1996). 